THURSDAY, JANUARY 26, 1871

\section{PHYSICAL LABORATORIES}

$\mathrm{T}$ is well known that chemistry can be taught far better 1 by a laboratory in which the student performs the various experiments, than by any system of lectures. Now, although for many years physicists have been in the habit of instructing their special students and assistants in this way, yet it is only recently that the same plan has been tried with large classes in physics. One of the first institutions to attempt this method, in America at least, was the Massachusetts Institute of Technology in Boston; and as I find many colleges here establishing physical laboratories, I trust that our experience may prove of some interest. The great difficulty is to enable twenty or thirty students to perform the same experiment without duplicating the apparatus, and to avoid the danger of injury to delicate instruments. Our plan is this:-Two large rooms (one nearly a hundred feet in length) are fitted up with tables, supplied with gas and water, somewhat like a chemical laboratory. On each is placed the apparatus prepared for a single experiment, which always remains in this place, thus avoiding the danger of breaking it in moving. A full written description is also given of each experiment, pointing out the proper precautions to avoid error or breakage. Near the door is an indicator or board containing the names of the experiments, and opposite each is placed a card bearing the name of the student. When the class enters the laboratory, they go to the indicator, and each member notices what experiment is opposite his name; he then goes to the proper table, reads the description, and performs it. He next reports his results to the instructor in charge, and if they are correct, his card is moved to some unoccupied place, and he proceeds as before. Care is taken that the number of experiments shall exceed that of students, and there is therefore no delay. The instructor in the mean time is enabled to pass from student to student and to see that no errors are committed. As quantitative work is far more valuable than qualitative, most of our experiments are of the former kind, and the student learns to measure physical constants and to verify laws numerically. For example, in one experiment a steel bar is supported on knife edges, and a weight is applied at the centre. The flexure is then measured by a micrometer screw, the exact point of contact being determined by including the screw and bar in the circuit of a battery and galvanometer. After making a number of experiments with various weights, the student constructs a curve in which ordinates represent deflection, and abscissæ weights applied. The law of elasticity shows that this curve should be a straight line, and the close agreement is convincing proof to the student of its correctness. In the same way the law of the conjugate foci of lenses is tested, and the observed curve compared with that deduced from theory. Some experiments are introduced to accustom the student to general methods of research, such as the computation of probable error by least squares, various forms of interpolation, \&c. The graphical method is largely used, as it at the same time enables the student to take in all his observations at one glance, while the instructor can constantly tell how vOL. III. carefully the work has been done. For the microscope a few objects are selected to show certain general methods of using this instrument, as one requiring a diaphragm, a second oblique illumination, and so on. Again, the student views by polarised light such objects as unannealed glass, crystals, designs in selenite, and studies the effects produced by various agencies. By thus handling the instruments he acquires a facility in using them and comprehension of their construction which he could never obtain from lectures. The excellence of the work done by many of the students led to the hope that valuable results might be attained by assigning to different students the experiments in a research, taking care that each should be repeated several times by different individuals. These results, if concordant, would be much more conclusive than those obtained by a single experimenter, since they would be free from all personal bias. In this way some interesting results have been attained on the foci of lenses placed obliquely, the flow of air through straight and curved tubes, and other similar subjects. Photometry and electrical measurement seem especially suited to this purpose, and the application of the latter subject to submarine cables would be both interesting and instructive to the student. During the winter time of 1869 and 1870 about sixty students worked in one laboratory, so that the experiment was tried on a sufficiently large scale to enable us to speak with confidence of its success. We found the system described above worked well, the students were interested in the subject, and obtained results of considerable accuracy. The loss by breakage was exceedingly small, and the current expenses insignificant compared with a chemical laboratory, since there is but little consumption of the materials employed.

There are now in America at least four similar laboratories either in operation or preparation, and the chances are that in a few years this number will be greatly increased. The value of a knowledge of physical manipulation is becoming daily better appreciated, and it is evident that instruction of this kind can be properly given only in a physical laboratory.

EDWARd C. PICKERING

SCIENCE TEACHING IN PRIVATE SCHOOLS

$A$ WRITER of the early part of last century defined a philosopher as one "whose trade was to do nothing, and to speculate upon everything." While philosophers were so jightly esteemed, it is no matter of surprise that philosophy was little cared for as a part of education. But such a definition as the above would not now be generally accepted even by the unscientific public. All are beginning to see that it is to Science they are indebted for so many of the comforts and advantages of civilisation, yet to the many is Science a mystery and closed book. And one great cause of this we believe to be, because it is not taught in our schools.

We purpose, in the present article, to speak only of private schools. If a visitation were to be made of such schools in England, we venture to say that very few, comparatively, would be found, in which Science, in any of its branches, is made a subject of regular education. The boys of most schools would be classed by the masters 